\title{
A TECHNICAL NOTE
}

\section{User and System Interface Issues in the Purchase of Imaging and Information Systems}

\author{
Steve Langer and Jihong Wang
}

\begin{abstract}
We introduce a set of worksheets to facilitate standardized comparisons among scheduling systems, hospital information systems, radiology information systems, and picture archiving and communication systems vendors. For each system category, we provide worksheets to evaluate the features, performance, installation requirements and costs of the included components. These worksheets will help to assure that critical user and systems interface issues are not overlooked and aid potential purchasers to make informed and objective purchasing decisions.

Copyright 11996 by W.B. Saunders Company
\end{abstract}

KEY WORDS: hospital information systems (HIS), radiology information systems (RIS), picture archiving and communication system (PACS), evaluation computers, radiology.

A PICTURE archiving and communication system (PACS) is a complex ensemble of equipment that involves an archive, acquisition gateways to imaging devices, and image review workstations. However, a PACS becomes much more useful when it is networked to a radiology information system (RIS), hospital information system (HIS), and scheduling system (SS). With these extensions, the PACS becomes a resource that is available to the entire institution, and patient scheduling, report generation, and patient tracking are greatly simplified. Unfortunately, the additional interfaces required to link the SS-HIS-RIS-PACS chain make an already complex purchasing decision even more daunting.

To simplify the purchasing process, a systematic approach is needed that facilitates comparison, not only among products of the same type, but between the interfaces that will link disparate systems. In this way, today's PACS purchaser can consider features that will be needed to interface the PACS with an installed RIS or HIS, or in the future when additional funding becomes available for RIS or HIS purchases.

\section{MATERIALS AND METHODS}

We introduce a consistent approach that incorporates four forms (features, performance, installation require- ments, and costs) for each segment of the SS-HIS-RISPACS chain. Each form includes every component relevant to that information system and the component aspects that are germane to the form topic. In some instances, a vendor may integrate conceptually different functions (such as HIS and RIS processes) into one product. In this eventuality, one could assemble a forms superset from the union of two conceptual systems.

The forms are included as stand alone appendices (in reduced form) to facilitate photocopying. Because the installation requirements and cost forms are substantially equivalent for RIS, SS and HIS equipment, they are only explicitly listed in the RIS Appendix.

\section{RESULTS}

Some concerns are common for all systems. Security, for instance, should be available in some form to prevent unauthorized access to patient data. Multiuser operating systems have an inherent advantage in this area, because such machines have built in security. Applications built for single user operating must add their own security features-with variable success. Also, all data base servers should have uninterruptible power supply (UPS) protection in the event of a power failure. The capacity of the UPS should be sufficient to warn users that power has failed, write memory resident data to disk, and automatically shut down the computers without loss of patient data. Typically, these requirements can be met with a 15 minute capacity. Finally, all systems should include some self-diagnostics. For workstations this may simply include memory, networking, and display tests. For data base servers and archives, it should include the former plus backup logs, file access logs, power outage logs, and file system integrity checks. Hard copy output and digitizing systems should perform self-calibrations on

From the Department of Diagnostic Radiology, Mayo Clinic and Foundation, Rochester, MN.

Address reprint requests to Steve Langer, PhD, the Department of Diagnostic Radiology, Mayo Clinic and Foundation, 200 First St SW, Rochester, MN 55905.

Copyright 101996 by W.B. Saunders Company

0897-1889/96/0903-0003\$3.00/0 
power up to assure that resolution and contrast gradients are performing as designed.

PACS/Teleradiology. Historically, PACS and teleradiology workstations have been considered to be distinct product lines, often sold by different vendors. From a functional standpoint, however, both systems perform the same job and should be considered in a similar light. In fact, some vendors are now performing such an integration. ${ }^{1}$ For this reason, our PACS/ Teleradiology forms cover not only PACS workstations, but also teleradiology workstations, archives, digitizers, modems, and hard copy devices.

The size, number, brightness, and resolution of monitors has a large impact on the suitability of a PACS workstation for different practices. For instance, what would be an acceptable resolution for an ultrasound or nuclear medicine diagnostic monitor would be insufficient for bone work. ${ }^{2}$ For this reason, it is likely that monolithic PACS will evolve into mini-PACS specialized by practice. Nevertheless, it is useful if images from all practices are centrally archived, either physically on the same hardware, or logically via a data base, to ease the search task for HIS and other image client software and users.

The ability of the review software to automatically window and level images in a modality sensitive manner is also desirable for productivity reasons. The workstation operating system also has a large impact on user efficiency. Single user, single tasking computer operating systems may be sufficient for smaller practices, but the greater reliability, throughput and security of multiuser, multitasking operating systems make them more suited to practices with a large workload.

The archive is the heart of the PACS. It must collect images from the imagers and pass them on to the PACS workstations for review. After interpretation, it must store the images and maintain links with the RIS so that the proper patient textual data is bound to the appropriate images. Finally, the archive must answer image recalls from the RIS so that ad hoc HIS and PACS workstation study requests are honored. Increasingly, communication between the archive and imagers will be in digital image communication in medicine (DICOM), but at present many systems require some sort of gateway to interface this transaction. Nor is the word "DICOM" alone sufficient to assure that the archive can accept all types of DICOM images. One must examine the vendor's DICOM Conformance Statement, and ideally test on-site the ability of the archive to accept DICOM inputs from magnetic resonance imaging (MR) computed tomography (CT), computed radiography (CR), ultrasound and other imaging devices. Communication between the archive and RIS may take place in DICOM, Health Level 7 (HL7) or structured query language (SQL) and it is important to know if the RIS and archive are compatible. For maximum speed in delivering images to workstations, one should seek out archives that use the latest generation of networking technology such as Ethernet II (which operates at over $100 \mathrm{Mbps}$ ). Combined with on-the-fly lossless image compression (with decompression on the workstation side), effective transmission rates of 300 Mbps can be reached, meaning that an 8 Mbyte chest radiograph can be transmitted in 0.2 seconds versus 6.4 seconds on standard Ethernet. Also, for maximum performance, the archive should have a layered mass storage system with a fast redundant array of independent disks (RAID) for recently accessed images and optical or tape systems for long-term backup.

In institutions that intend to digitize an existing film archive, or will continue to perform some film exams but prefer soft copy diagnosis, film digitizers will be required. The most important feature to note is whether the full resolution is available at all film sizes or fields of view. Line pair resolution should exceed $31 \mathrm{p} / \mathrm{mm}$ and the digitizer should remain linear through a 3.0 optical density range. A $10 \sim 12$ bit $(1,024 \sim 4,096$ shades $)$ gray scale range is also recommended to represent the full range of data that may be in a radiograph. ${ }^{3}$

Workstations used in a teleradiology mode will require modems. These should be the highest speed that one can afford and ideally will communicate with a modem bank on the PACS so that images from regional sites are centrally archived.

In the electronic imaging environment, hard copy will become less and less used. However, when hard copy is desired, it should be provided from networked laser cameras, preferably by systems that use dry film technology (for mainte- 
nance and cost reasons). These cameras require gray scale and resolution performance similar to the film digitizer and should provide an optical density range of 2.5 (when used in an MR or CT mode) and 3.0 or higher when used for CR work. $^{4,5}$ It is also helpful if the units are selfcalibrating.

RIS. The RIS forms cover client workstations and data base servers. Because the RIS data base will act as the Radiology department's information system to the rest of the medical center, it is essential that it be able to communicate with the HIS, SS, billing information system, as well as the PACS. ${ }^{6}$ It should have data base facilities that allow for exam descriptions, including exam duration and preparation procedures, so that scheduling personnel can instruct the patient and avoid overbooking. The data base must also provide tools to retrieve studies via name, gender, diagnosis, exam type, age, race, occupation, exam date, and other identifiers to facilitate research, teaching and clinical care. Re-entry of patient demographic information is minimized if the RIS data base communicates directly with the imagers. For instance, if a patient is provided with a coded card on admission, the card need only be passed through a reader attached to the imager when the patient arrives for their exam. The imager then checks the patient identity against the RIS schedule and proceeds with the exam, or generates an error if an inconsistency is found.

From a workflow and cost viewpoint, the RIS client software would optimally run simultaneously on the PACS workstation and support a minimal image display. This would allow radiologist confirmation that the RIS patient entry matches the PACS study, and allow report generation (via typing or voice recognition) with minimal work disruption. More often, the RIS client runs on a separate computer and the radiologist (either alone or with the aid of a transcriptionist) must coordinate synchronizing the patient entry on the two systems.

HIS. The HIS forms cover client workstations and data base servers. Because HIS client workstations will be used by virtually all clinicians, they must be capable of displaying a wide variety of data including images, graphs and text. However, because there will also be a relatively large number of them, the costs per unit must be minimized as much as possible. A single large (17 inch) monitor of moderate brightness ( 80 foot Lamberts) and resolution $(1,000 \times 1,000$ or $1,000 \times 800)$ may be sufficient. ${ }^{2}$ Equally important is how best to present the necessary information on the screen. Ideally, a user would log into the system and a personal defaults file would be used to configure the screen layout. A patient query should produce an alphabetized display of all the exams the patient has had, plotted against a time-line. Selecting "Lab Results," for instance, should display which tests have been done or scheduled, and offer the option to display results. Choosing "Radiology Results" would similarly list completed and/or pending exams, and offer the text report with an option for image display.

The HIS data base acts as the coordinator of HIS workstation requests to the appropriate departmental information servers. As such, its mission is to be aware of the exams a patient has had (or will have) and construct the resulting list on the HIS workstation. Ideally, the HIS data base should also be capable of caching recent patient data in fast short-term storage to enhance performance.

SS. The SS forms cover workstation and data base units. The SS data base is the agent that defines what (and when) events will happen to the patient as opposed to the HIS data base which reports on events that have happened to the patient. The SS must have the ability to communicate with every departmental information system and be capable of negotiating exam times within the constraints imposed by each department. It should also share this information with the HIS to allow clinicians the opportunity to see all pending exams that are scheduled for their patients.

The SS client should display information in a manner similar to the HIS, but emphasize exam times and preparations rather than results. A daily or weekly planner, with free hours highlighted in one color and exams times in another, is often a helpful display.

\section{DISCUSSION}

The forms are included in reduced format in the appendices of this report. They are also available full size in Rich Text Format (a form supported by most modern word processors) for either DOS or Macintosh computers (Apple 
Computer, Cupertino, CA) from the anonymous ftp site:

ri-exp.beaumont.edu/pub/diag/Dos/

$$
\text { pac-bg.rtf or ri-exp.beaumont.edu/pub/ }
$$$$
\text { diag/Mac/pac-bg.rtf }
$$

Upon retrieval the forms display parameters in one column. In the second column, space is provided adjacent to each parameter for identifying the vendor, writing comments, and recording performance measurements. The forms delve deeply into specific attributes, including performance specifications, for each information system. By the completing the PACS forms (for example) and comparing them with completed RIS (or HIS or SS) forms, one can determine if the systems are compatible. Conversely, one can compare and contrast completed PACS forms from different vendors to assist in the purchasing decision for that specific system, or ascertain the compatibility of each practice's mini-PACS.

The worksheets can form the basis for a purchasing specification, but that is not the intent. If one has preconceived performance specifications or holds a vendor only to features that are written in these forms, one could actually inhibit vendor motivation for improvement or introduction of new functions. Furthermore, these forms in no way replace the detailed negotiations, which must occur when finalizing a purchasing decision. A detailed purchasing agreement (which specifies what is to be bought and at what price, methods of payment, acceptance testing criteria, warranty and service considerations) must still be used. ${ }^{7}$ Rather, these forms should be used to facilitate high level coordination and comparison of critical features that should not be missed in a purchasing decision, and to standardize comparisons of basic functions and performance that are common among vendors in the electronic medical record marketplace.

Identifying user and system interface compatibility issues is critical for maximizing user productivity and assuring that the various information systems in a modern medical center communicate efficiently. A systematic approach can assist the purchaser in weighing the various alternatives, and help avoid costly mistakes in implementing, using and maintaining such systems.

\section{APPENDIX 1: PACS AND TELERADIOLOGY}

\section{Features}

\section{Parameter}

Workstation (vendor). Number of monitors (1, 2 or more); Monitor size and curvature; Monitor resolution $(\mathrm{lp} / \mathrm{mm}$, modulation transfer function [MTF], line spread function); Monitor brightness (uniformity, flicker); Operating System/Interface, security; Manual Window/ Level; Automatic, Modality Smart Window/ Level; Teaching file support (slide making, special directories); Computer aided diagnosis tools (neural net, expert system); Image tools (volume render, signal to noise ratio, contrast to noise ratio, and region of interest (ROI) measurement, multiimage registration, edge enhancement, filters, and kernels); Personal user defined preferences; Cine mode; On the fly image decompression; Remote maintenance capability; DICOM I/O; Self-diagnostics

Archive (vendor). Fast short-term storage (hard drive, RAID); Long-term storage (optical disk jukebox [ODJ], tape); Direct DICOM inputs (or gateways); Direct DICOM outputs (or gateways); Internal image data base (HL7, DICOM, SQL); RIS-HIS Commun. (HL7, DICOM, SQL); Automatic UPS safe shutdown; On the fly image compression; Support for modem access; Security, remote maintenance, disaster recovery; Self-diagnostics

Digitizer (vendor). Autosizing; Resolutions; Full resolution available at any size; MTF, maximum bit depth; Operating system/interface; Auto-send; Compression; Remote maintenance capability; DICOM I/O; Self-diagnostics

Modems (vendor). Type (Vbis 32, internal, external)

Hard copy output (vendor). Self-calibration (sensi strip, Society of Motion Pictures and Television Engineers); Film or paper (dry/wet film, dye sublimation); Resolution

\section{Performance}

\section{Parameter}

Workstation. Patient list retrieve speed (sec); Study retrieve speed (normed to Mbyte/sec); Time to Display first study image; Screen paint time (normed to Mbyte/sec); Volume render speed (normed to Mybte/sec) 
Archive. Short-term capacity (Mbyte, days); long-term capacity (Mbyte, days); Max. number of imager inputs; Max. number of simulation outputs; Single connect archive speed (Mbyte/ sec); Single connect transmit speed (Mbyte/ sec); percent uptime, mean time before failure

Digitizer. Throughput/hr $(14 \times 17,8 \times 10)$; Transmit speed (Mbyte/sec include compress)

Modems. Transfer speed (Baud with error correction)

Hard copy output. Optical density range; Throughput per hour ( 1 on 1,12 on 1$)$

\section{Requirements}

\section{Parameter}

Environment. Workstation (temperature, humidity); Archive (temperature, humidity); Digitizer (temperature, humidity); Hard copy (temperature, humidity)

Power. Workstation (volt, amp, stability); Archive (volt, amp, stability); Digitizer (volt, amp, stability); Hard copy (volt, amp, stability)

Network. PAC workstations (Ether, Token or fiber distributed data interface [FDDI]); Archive (Ether, Token or FDDI); Tele workstations (Integrated services digital network [ISDN], Asynchronous transfer mode, T1, T3); Hard copy (networked or wired to central processing unit [CPU]

UPS. Archive (15 minutes capacity)

\section{Costs}

\section{Component}

Workstations. per Monitor, CPU; per software license; Service contract (warranty, training, upgrades, maintenance)

Archive. Short-term (RAID) plus CPU; Long-term unit (ODJ, tape) plus CPU; license fee per node; service contract (warranty, training, upgrades); UPS

Digitizer. Digitizer and associated CPU; per software license; service contract (warranty, training, upgrades)

Modems. Modem price; line lease charge (per month)

Hard copy unit. Unit costs; media (paper or film) costs

\section{APPENDIX 2: RIS}

\section{Features}

\section{Parameter}

Workstation (vendor). Monitor size and resolution; PACS interface (SQL, HL7, DICOM); Operating System/Interface, security; Capable of running on PACS workstation; Support for report generation (keyboard or voice dictation); Self-diagnostics

Data base Server (vendor). Operating Systems/interface; HIS-SS-PACS interface (HL7, DICOM, SQL); Safe UPS shutdown; Security, remote maintenance, disaster recovery; Keyboardless patient data transfer (bar-code download to imager?)

\section{Performance}

\section{Parameter}

Workstation. Patient List Retrieve (sec); Data base lookup (sec); Patient record retrieve (sec)

Data base Server. Max. number of simulated connections; transactions per second; percent uptime, mean time to failure

\section{Requirements}

\section{Parameter}

Environment. Workstations (temperature, humidity); data base server (temperature, humidity)

Power. Workstations (amp, voltage, stability); data base server (amp, voltage, stability)

Network. Workstation (Ethernet, Token Ring, FDDI); data base server (Ethernet, Token, FDDI)

UPS. Data base server (15 min capacity)

\section{Costs}

\section{Component}

Workstation. CPU and monitor; software license per node; Service contract (warranty, training, upgrades)

Data base Server. CPU and storage; software license per node; service contract (warranty, training, upgrades); UPS 


\section{APPENDIX 3: HIS}

\section{Features}

\section{Parameter}

Workstation (vendor). Operating system/ Interface, security; Screen size, resolution, brightness (lp/mm, MTF); PACS-RIS and other IS interface (HL7, Massachusetts general multiprogramming system [MUMPS], DICOM, SQL); Image, text, and graph displays; Image tools (cine, win/lvl, magnify, ROI); On the fly image decompression; Point of care data entry; Modem capability; Remote maintenance capability; Self-diagnostics

Data base server (vendor). Operating systems/interface; Interface with other IS (HL7, DICOM, SQL, MUMPS); Modem capability for off-site HIS workstation; Safe UPS shutdown; Security, remote maintenance, disaster recovery

\section{Performance}

\section{Parameter}

Workstation. Patient data base search (sec); Study retrieval time (normed to Mbyte/sec); Screen paint time (normed to Mbyte/sec); Volume render speed (normed to Mbyte/sec)

Data base server. Max. number of simulated connections; transactions per second; percent uptime, mean time to failure

\section{APPENDIX 4: SS}

\section{Features}

\section{Parameter}

Workstation (vendor). Operating system/ Interface, security; Screen Size, resolution, brightness; PACS-RIS and other IS interfaces (HL7, MUMPS, DICOM, SQL); Cooperate with billing, RIS and other IS for exam preparation; Cooperate with HIS to show exams scheduled but not completed; Remote maintenance capability; Self-diagnostics

Data base server (vendor). Operating Systems/interface; Interface with other IS (HL7, DICOM, SQL, MUMPS); Safe UPS shutdown; Security, remote maintenance, disaster recovery

\section{Performance}

\section{Parameter}

Workstation. Patient list lookup (sec); Patient status data base query (sec); Time to schedule appointment ( $\mathrm{sec})$

Data base server. Max. number of simulated connections; transactions per second; percent uptime, mean time to failure

\section{REFERENCES}

1. Siemans Medical Engineering: MagicView 50. Erlangen, Germany, 1996

2. Dwyer S, Stewart B: Clinical uses of grayscale workstations. AAPM Summer School Proceedings 243-262, 1993

3. Trueblood JH, Burch SE, Kearfott K, et al: Radiographic film digitization. AAPM Summer School Proceedings 99-122, 1993

4. Gray JE, Anderson WF, Shaw CC, et al: Multiformat video and laser cameras: History, design considerations, acceptance testing and quality control. Report of AAPM
Diagnostic X-Ray Imaging Committee Task Group No. 1. Med Phys 20:427-438, 1993

5. Siegel EL, Bishop MD, Wetzal LH, et al: Hardcopy recording technologies for digitally formatted image data. Invest Radiol 26:179-183, 1991

6. Hayes E: Systems Integration: Information needs soar in managed care. Diagn Imaging September: 24-27, 1995

7. Gray JE, Morin RL: Purchasing medical imaging equipment. Radiology 171:9-16, 1989 